CERN-PPE/95-174

13 October 1995

\title{
On the electromagnetic energy resolution of Cherenkov-fiber calorimeters
}

\author{
M. Lundin ${ }^{1, a)}$, A. Contin ${ }^{2)}$, G. Dellacasa ${ }^{3, b)}$, R. DeSalvo ${ }^{4)}$, M. Gallio ${ }^{3)}$, \\ P. Gorodetzky ${ }^{1,5)}$, J.M. Helleboid ${ }^{1)}$, K.F. Johnson ${ }^{6)}$, P. Juillot ${ }^{1)}$, D. Lazic ${ }^{6, c)}$, A. Musso ${ }^{3)}$, \\ E. Vercellin ${ }^{3)}$, S. White ${ }^{7}$
}

\begin{abstract}
Electromagnetic calorimeters which sample the Cherenkov radiation of shower particles in optical fibers operate in a markedly different manner from calorimeters which rely on the $\mathrm{dE} / \mathrm{dx}$ of shower particles. The well-understood physics of electromagnetic shower development is applied to the case of Cherenkov-fiber calorimetry (also known as quartz fiber calorimetry) and the results of systematically performed studies are considered in detail to derive an understanding of the critical parameters involved in energy measurement using such calorimeters. A quantitative parameterization of Cherenkov-fiber calorimetry electromagnetic energy resolution is proposed and compared with existing experimental results.
\end{abstract}

(To be published in Nuclear Instruments and Methods in Physics Research)

1) CRN and Université Louis Pasteur, Strasbourg, France

2) University of Bologna and INFN Bologna, Italy

3) University of Torino and INFN Torino, Italy

4) INFN Pisa, Italy

5) CERN, Geneva, Switzerland

6) Florida State University, Tallahassee, FL, USA

7) Brookhaven National Lab, Upton, NY, USA

a) Corresponding author, presently at Ames Laboratory and Department of Physics, Iowa State University, Ames IA, USA

b) Presently at II Faculty of Science of Torino University, Alessandria

c) Presently at Israel Institute of Technology (Technion), Haifa, Israel 

The increasingly demanding requirements imposed on calorimeters in both fixed target and collider mode for existing and future heavy-ion and hadron accelerators has lead to the relatively recent development of the quartz fiber calorimetry (QCal) technique [1-20]. The primary characteristics of interest for such calorimeters are the impressive potential for the radiation hardness of amorphous silica $[3,15,21]\left(\mathrm{SiO}_{2}\right.$, often referred to simply as "quartz"), the intrinsic speed of a device which relies on Cherenkov-effect light for information and the small effective shower radius $[15,6,19]$.

The quartz fiber calorimeter is based on optical fibers, acting as the calorimeter sampling material, embedded in high $\mathrm{Z}$ absorber material to form a sampling calorimeter which is sensitive only to the Cherenkov light radiated by shower particles. Information about the shower (in the form of various wavelengths of light) is transmitted through the same optical fibers. Although the use of radiation-hard quartz optical fibers is at the root of present interest in this calorimetry technique, inexpensive plastic optical fibers are also suitable for calorimetry in low radiation dose environments, provided they emit no scintillation light. We have performed a systematic study involving prototypes loaded with various types of fibers (both silica and plastic based) and so will refer to this calorimetry technique as Cherenkov-fiber calorimetry in the following discussion.

Optimized detector design comes through the understanding of both cascade processes and the numerous instrumentation variables and calorimeter characteristics. A clear and accurate understanding of the elementary processes behind electromagnetic shower development has existed for some time. Here we attempt to quantitatively define the effects of the most important detector characteristics on overall performance. Our attempts are heavily influenced by the seminal work of a number of authors [22-25], primarily U. Amaldi [25], whose lucid explanations form a solid base for any such discussion. We therefore begin from existing and generally accepted parameterizations and make alterations based on our own understanding of the Cherenkov-fiber calorimeter.

In section 2 the characteristics of existing Cherenkov-fiber prototypes are described. In section 3 we discuss the causes of energy mis-measurement and go on to parameterize Cherenkov-fiber calorimeter energy resolution based on a simple phenomenological model. In section 4 we compare the results of our parameterization with experimental results, followed by conclusions in section 5 .

\section{The Cherenkov-fiber calorimeter}

\subsection{General design considerations}

Seven slightly different prototypes were constructed with fibers arranged in ribbons (sandwiched between plates of absorber material) with no transverse separation between fibers. The seven prototypes are reported in detail in references [11, 15, 19] and are only briefly described here. All prototypes had the common characteristic that their optical fibers and absorber plates were arranged at $45^{\circ}$ with respect to incoming particles. This angle (being at or close to the Cherenkov angle) was found to maximize detector response in terms of collected Cherenkov photons. More in-depth discussions on this geometrical choice can be found in references $[15,19,20]$. This fiber arrangement, a quasi-uniform lateral distribution of fibers, was shown to improve energy resolution over a staggered or matrixed arrangement [15]. We attribute this gain in energy resolution to the improved sampling of showers in their early stages, where the core is extremely narrow and dense. The pertinent characteristics of the seven prototypes are given in Table 1. 
Table 1: Characteristics of the electromagnetic Cherenkov-fiber calorimeter prototypes.

\begin{tabular}{|c|c|c|c|c|c|}
\hline Prototype & $\begin{array}{c}\text { Fiber Type } \\
\text { Core/Clad/Coat } \\
\text { Diameter }(\mu m)\end{array}$ & $\begin{array}{c}\text { Fiber } \\
\text { NA }\end{array}$ & $\begin{array}{c}\text { Absorber } \\
\text { Type } \\
\text { and } \\
\text { Thickness }\end{array}$ & $\begin{array}{c}\text { Volume } \\
\text { Filling } \\
\text { Ratio } \\
\text { (Abs.:Fib.) }\end{array}$ & $\begin{array}{c}X_{o} \\
(\mathrm{~cm})\end{array}$ \\
\hline Proto- $\mathrm{Cu} 1.5$ & $\begin{array}{l}486 \mu m \text { PMMA/ } \\
500 \mu m \text { PMMA }\end{array}$ & 0.51 & $1.5 \mathrm{~mm} \mathrm{Cu}$ & $4.0: 1$ & 2.0 \\
\hline Proto- $\mathrm{Cu} 3$ & $\begin{array}{l}486 \mu m \text { PMMA/ } \\
500 \mu m \text { PMMA }\end{array}$ & 0.51 & $3 \mathrm{~mm} \mathrm{Cu}$ & 8.1:1 & 1.7 \\
\hline Proto-Pb3 & $\begin{array}{c}486 \mu \mathrm{m} \text { PMMA/ } \\
500 \mu \mathrm{m} \text { PMMA }\end{array}$ & 0.51 & $3 \mathrm{~mm} \mathrm{~Pb}$ & $8.1: 1$ & 0.65 \\
\hline Proto-3 & $\begin{array}{c}500 \mu m \mathrm{SiO}_{2} / \\
540 \mu m \mathrm{HC}\end{array}$ & 0.37 & $3 \mathrm{~mm} \mathrm{~Pb}$ & $8.3: 1$ & 0.67 \\
\hline Proto- 4 & $\begin{array}{c}500 \mu \mathrm{m} \mathrm{SiO}_{2} / \\
550 \mu \mathrm{m} \text { silicone/ } \\
600 \mu \mathrm{m} \text { acrylate }\end{array}$ & 0.4 & $3 \mathrm{~mm} \mathrm{~Pb}$ & $9.2: 1$ & 0.67 \\
\hline Proto-R & $\begin{array}{c}500 \mu \mathrm{m} \mathrm{SiO} / \\
530 \mu \mathrm{m} \mathrm{SiO}(F) / \\
560 \mu m \text { polyimide }\end{array}$ & 0.22 & $3 \mathrm{~mm} \mathrm{~Pb}$ & $8.6: 1$ & 0.67 \\
\hline Proto- $\mathrm{Cu} 20$ & $\begin{array}{c}486 \mu m \text { PMMA/ } \\
500 \mu m \text { PMMA }\end{array}$ & 0.51 & $20 \mathrm{~mm} \mathrm{Cu}$ & $54: 1$ & 1.5 \\
\hline
\end{tabular}

Transverse dimensions of the front faces of the seven electromagnetic prototypes were $5 \times 5 \mathrm{~cm}^{2}$ and lengths of the prototypes varied so as to fully contain high energy electron showers. The calorimeters were laterally divided into two independent, geometrically seamless, readout channels as graphically depicted through the schematic diagram of Proto-3 shown in Fig. 1. Each detector half was fully shower containing for electrons impinging at its center. Readout of the prototypes was performed using UV sensitive, Philips XP2020/Q phototubes (Bialkali-D photocathode) via air core, hexagonal light mixers.

\subsection{Choice of optical fiber}

Prototypes were filled with optical fibers whose core diameters were close or equal to $500 \mu \mathrm{m}$. Different types of fiber were used to measure the influence of the fibers' Numerical Aperture ${ }^{1)}$ (NA) on calorimeter response. Fiber cores consisted of both amorphous silica (Proto-3, Proto-4 and Proto-R) and PolyMethyl MethAcrylate (PMMA) (Proto$\mathrm{Pb} 3$, Proto $\mathrm{Cu} 3$, Proto-Cu1.5 and Proto-Cu20). Cladding materials varied in thickness and type. The fibers used for Proto- 3 were manufactured by CeramOptec ${ }^{2)}$ and their cladding consisted of a proprietary plastic, "hard cladding" (HC) material. Another type of CeramOptec fiber was used for Proto-4, and its silicone cladding was surrounded by an additional protective acrylate coating. The coating material is part of a mechanical protection as the fiber was intended by the manufacturer to be used for optical data transmission. The coating performs no function in terms of the fiber light guide, although

1) Numerical aperture is defined through the refractive indices of fiber core and cladding material: $N A=\sqrt{n_{\text {core }}^{2}-n_{\text {clad }}^{2}}$.

2) CeramOptec GmbH, Bonn, Germany 


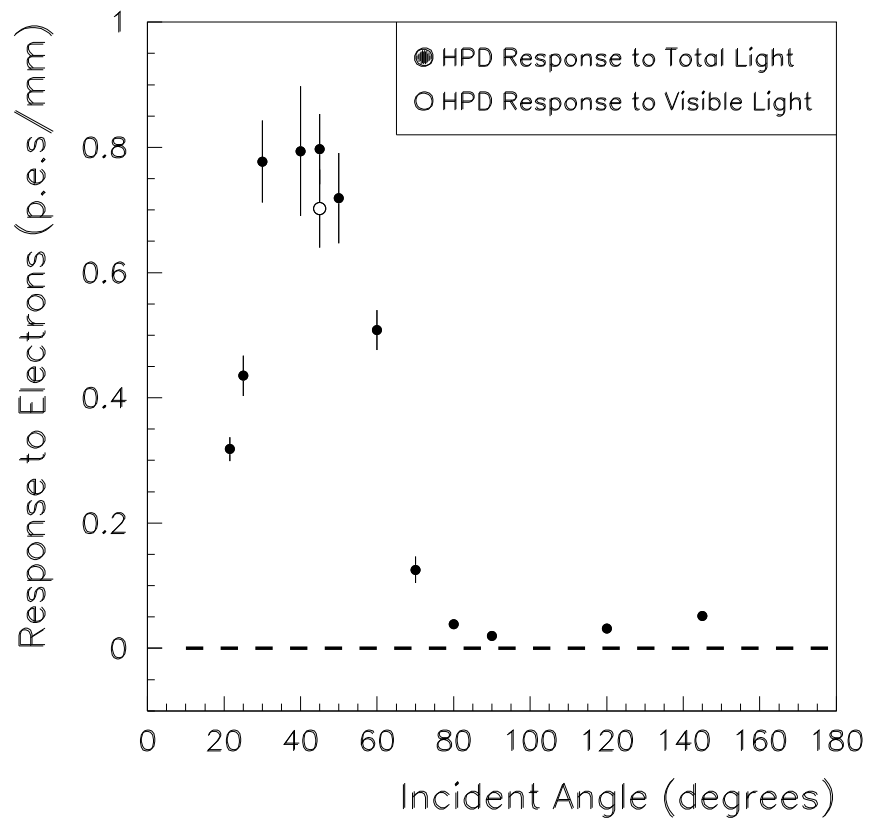

Figure 2: Mitsubishi PMMA core with PMMA cladding, 1.5mm diameter fibers' response to $8 \mathrm{GeV}$ single electrons as a function of incident angle.

combine to result in roughly the same calorimeter response. In environments where fibers will be subjected to low doses, plastic core, plastic clad, non-scintillating fibers might be used. This could conceivably become one of the least expensive of available calorimetry techniques, since clear plastic fibers are among the cheapest of sampling media.

\subsection{Measured response to electrons}

Beam tests were performed using 5 to $100 \mathrm{GeV}$ electrons on the electromagnetic prototypes Proto-3, Proto-4 and Proto- $\mathrm{R}$ while all others were tested with energies ranging from 2 to $120 \mathrm{GeV}$. Tests on all prototypes were performed at the CERN PS and SPS during 1994 and 1995. Fig. 3 shows each calorimeter's electron energy resolution as a function of $1 / \sqrt{E(G e V)}$. Measured energy resolutions were fit in quadrature according to Equation (1):

$$
\frac{\sigma(E)}{E}=\sqrt{\left(\frac{a^{2}}{E}+b^{2}\right)}=\frac{a}{\sqrt{E}} \oplus b,
$$

where we follow convention by referring to $a$ as the energy resolution stochastic term and to $b$ as the energy resolution constant term. Cherenkov-fiber calorimeters have the unique characteristic of being sensitive only to the rapid and forward moving particles of the shower core $[15,19]$ and the prototypes discussed here were measured to have transverse shower widths typically $\sim 4$ times smaller than that of typical $\mathrm{dE} / \mathrm{dx}$ based calorimeters (for $95 \%$ shower containment [19]). The energy resolution for each prototype was measured using single channels (each channel being a fully shower containing detector half) and also with the sum of both readout channels. In all cases results were similar but, as only $1 / 2$ of each detector was sufficient for lateral shower containment, the measured energy resolution for only one readout channel is shown in Fig. 3. 


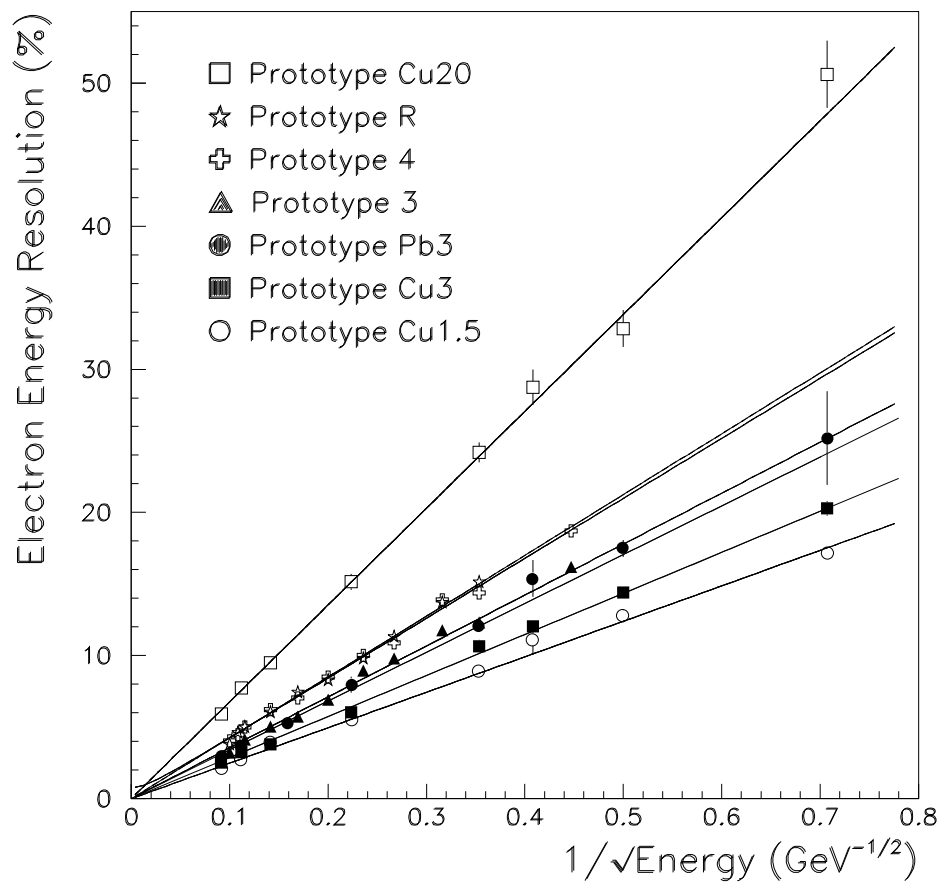

Figure 3: Quadrature fits to the energy resolution of the seven electromagnetic Cherenkov-fiber prototypes. Energy resolution constant terms compatible with 0 are evident in all cases.

Electromagnetic energy resolution stochastic terms varied depending on the frequency of sampling material in a given calorimeter prototype. Each prototype was measured to have an energy resolution constant term compatible with 0 . This significant result should be contrasted with other geometries involving fiber matrixing resulting in considerable energy resolution constant terms $[16,19]$. The results of fits are shown in Table 2 along with the measured number of photoelectrons per Minimum Ionizing Particle (MIP) (estimated using muon crossings) and the measured number of photoelectrons per $\mathrm{GeV}$ of incident energy. Descriptions of photoelectron light output measurements can be found in references $[11,15,19]$.

\section{Causes of energy mis-measurement}

Photoelectron statistical fluctuations and sampling fluctuations account for a major portion of degradation in the energy resolution of Cherenkov-fiber calorimeters. The amount of signal from the Cherenkov effect in optical fibers is of the order of tens of photoelectrons per $\mathrm{GeV}$ of incident energy (depending on the calorimeter filling ratio) and small fluctuations in response on this signal level correspond to a large fraction of the total signal at lower energies. Landau fluctuations also play a role, but this effect is significant only for very low sampling fraction calorimeters.

Measurements performed on the seven comparable prototype calorimeters reveal a more or less equal contribution from sampling fluctuations and photoelectron statistical fluctuations in each case. The summarization of energy resolutions in Table 1 allows conclusions to be drawn about the stochastic term for geometries in which the constant term is compatible with 0 . The discussion which follows is guided by the following basic ideas:

- Sampling fluctuations are a major source of energy resolution degradation in any 
Table 2: Measured light production characteristics and electromagnetic energy resolutions for the electromagnetic Cherenkov-fiber calorimeter prototypes. While depositing only $\sim 300 \mathrm{MeV}$ in the detector, muons produce 0.6 to $0.8 \mathrm{GeV}$ of equivalent energy and can therefore be used for calibration purposes.

\begin{tabular}{|c|c|c|c|c|}
\hline Prototype & $\begin{array}{c}N_{\text {p.e. }} / M I P \\
\text { (muons) } \\
\text { UV } \\
+ \\
\text { Visible }\end{array}$ & $\begin{array}{c}N_{\text {p.e. }} / \mathrm{GeV} \\
\text { (electrons) } \\
\mathrm{UV} \\
+ \\
\text { Visible }\end{array}$ & $\begin{array}{c}\text { EM Energy } \\
\text { Resolution } \\
\text { Constant } \\
\text { Term }\end{array}$ & $\begin{array}{c}\text { EM Energy } \\
\text { Resolution } \\
\text { Stochastic } \\
\text { Term }\end{array}$ \\
\hline Proto- $\mathrm{Cu} 1.5$ & $46.6 \pm 2.0$ & $57.7 \pm 2.7$ & $(0 \pm 1.4) \%\left(^{a}\right)$ & $(24.8 \pm 0.3) \%\left(^{a}\right)$ \\
\hline Proto-Cu3 & $23.1 \pm 1.0$ & $29.3 \pm 1.5$ & $(0 \pm 0.5) \%\left(^{a}\right)$ & $(28.7 \pm 0.3) \%\left(^{a}\right)$ \\
\hline Proto-Pb3 & $7.85 \pm 0.69$ & $13.9 \pm 1.6$ & $(0 \pm 0.4) \%\left({ }^{a}\right)$ & $(34.1 \pm 0.3) \%\left(^{a}\right)$ \\
\hline Proto-3 & $9.0 \pm 0.6$ & $15.9 \pm 1.6$ & $(0 \pm 0.4) \%\left(^{b}\right)$ & $(35.6 \pm 0.2) \%\left(^{b}\right)$ \\
\hline Proto-4 & $9.9 \pm 0.6$ & $12.8 \pm 1.5$ & $(0.7 \pm 0.6) \%\left(^{b}\right)$ & $(41.7 \pm 0.3) \%\left(^{b}\right)$ \\
\hline Proto- $\mathrm{R}$ & $12.6 \pm 1.0$ & $16.3 \pm 1.7$ & $(0 \pm 1.1) \%\left(^{b}\right)$ & $(42.3 \pm 0.7) \%\left(^{b}\right)$ \\
\hline Proto- $\mathrm{Cu} 20$ & $3.5 \pm 0.15$ & $4.9 \pm 1.0$ & $(0 \pm 1.0) \%\left({ }^{a}\right)$ & $(67.7 \pm 0.9) \%\left(^{a}\right)$ \\
\hline
\end{tabular}

sampling calorimeter. This effect is directly related to the path length of shower particles in a calorimeter's sampling material; resolution improves as the square root of sampling material frequency.

- Photoelectron statistics are a substantial limiting factor in the case of the Cherenkovfiber technique.

- Although the mean values of these two effects may be correlated, variation of calorimeter response due to each is uncorrelated. Therefore, fluctuations in calorimeter response caused by variance of the number of photoelectrons measured and by variance in the number of shower tracks sampled should be used to arrive at an energy resolution stochastic term by directly adding Gaussian variances $\left(\sigma^{2}\right)$ or by adding energy dependent resolutions $(\sigma / E)$ in quadrature [26].

\subsection{Photoelectron statistics fluctuations}

Fluctuations in photoelectron statistics can be related to the response of the calorimeter in photons/ GeV multiplied by the response of the photon readout device in photoelectrons/photon. Variables involved include the absorber material used, photon radiation through the Cherenkov effect, the fiber numerical aperture (light capturing power), transmittance of the core and cladding material as a function of wavelength, optical coupling at the fiber readout end and quantum efficiency of the light detectors used. The contribution to energy resolution degradation due to statistical fluctuations in the number of photoelectrons detected can be given as:

$$
\left(\frac{\sigma(E)}{E}\right)_{p e}=\sqrt{\frac{1}{\left.<n_{p e}\right\rangle}},
$$

where $n_{p e}$ is the number of photoelectrons per $\mathrm{GeV}$ of deposited energy. All measurements presented here were performed with phototubes operating at high gain, therefore effects of fluctuations at the dynode and collecting surface of the photodetector are safely neglected in Equation (2). These statistical fluctuations can be a substantial component of the energy resolution for small values of $\left\langle n_{p e}\right\rangle$. 
Table 3: Estimation of the degradation of electromagnetic energy resolution due to photoelectron statistic fluctuations as calculated using Equation (2).

\begin{tabular}{lc}
\hline Prototype & $\begin{array}{c}\left(\frac{\sigma(E)}{E}\right)_{\text {p.e. statistics }} \\
(\% / \sqrt{E(G e V)}) \\
\end{array}$ \\
& eq. $(2)$ \\
\hline Proto-Cu1.5 & $13.2 \pm \mathbf{0 . 6}$ \\
Proto- $\mathrm{Cu} 3$ & $18.5 \pm 0.9$ \\
Proto-Pb3 & $26.8 \pm 3.1$ \\
Proto-3 & $25.1 \pm 2.5$ \\
Proto-4 & $28.0 \pm 3.2$ \\
Proto- $\mathrm{R}$ & $24.8 \pm 2.5$ \\
Proto- $\mathrm{Cu} 20$ & $45.2 \pm 9.2$ \\
\hline
\end{tabular}

Contributions to electromagnetic energy resolution from photoelectron statistical fluctuations for quartz and plastic fiber calorimeter prototypes were computed by measuring the number of photoelectrons per $\mathrm{GeV}$ [11] for each and applying Equation (2). Results of this calculation, using the information from Table 2, for each of the seven prototypes described in section 1 are shown in Table 3. Photodetector gains were typically on the order of $10^{5}$ to $10^{6}$ and only one readout channel was used in the determination of energy resolution.

\subsection{Sampling fluctuations}

Previous authors $[27,25,28]$ have identified the frequency of sampling material as a critical variable in $\mathrm{dE} / \mathrm{dx}$ based calorimeters. An attempt at quantifying energy mis-measurement due to sampling fluctuations for Cherenkov-fiber calorimeters is proposed here. The discussion which follows is guided by the work done by Amaldi [25] for $\mathrm{dE} / \mathrm{dx}$ based calorimeters. Amaldi's arguments are briefly described and, where necessary, changes are made for the Cherenkov-fiber calorimetry case. It should be stressed that the following discussion refers only to those sampling calorimeters whose absorber and sampling materials are separated into alternating layers. This frequency of shower sampling can be expressed, in terms of radiation lengths per absorber-sampling material layer, by:

$$
t=x / X_{\circ}
$$

where $x$ (in units of $\mathrm{cm}$ ) is the distance between equally spaced absorber/sampling material layers and $X_{\circ}$ (in units of $\mathrm{cm}$ per radiation length) is the effective radiation length of the calorimeter, calculated as a homogenous mixture of materials. The energy measurement error due only to sampling fluctuations is then:

$$
\left(\frac{\Delta E}{E}\right)_{\text {sampling }}=R(\%) \sqrt{\frac{t}{E(G e V)}},
$$

where $R$ is a constant. Amaldi computes $R$ [25] by beginning with the assumption that the magnitude of sampling fluctuations is dependent on the total number of electron and positron tracks crossing sensitive planes. This number can be approximated by first considering the total track length, $T$, for an electromagnetic shower. Assuming that a 
given calorimeter is sensitive to all tracks in a shower regardless of shower particle energy (cut-off energy, $E_{c}=0$ ), this quantity would be expressed as:

$$
T=\frac{E X_{o}}{\epsilon},
$$

where $\mathrm{E}$ is the energy of the incident particle in $\mathrm{GeV}, X_{o}$ is the calorimeter's radiation length and $\epsilon$ is the electron critical energy for a given material. Equation (5) states that total track length is directly proportional to the energy of the incident particle. $\epsilon$ is defined as in Rossi's "Approximation B" [22], in which all electrons lose a constant amount of energy $(\epsilon)$ per radiation length of material crossed. Rossi's definition motivates the total sum of shower tracks lengths (as given in Equation (5)) being inversely proportional to $\epsilon$. We calculate the electron critical energy as a function of the absorber material atomic number: $\epsilon=610 /(Z+1.24)(7.3 \mathrm{MeV}$ and $20.2 \mathrm{Mev}$ for $\mathrm{Pb}$ and $\mathrm{Cu}$, respectively) as suggested through fits of available experimental data for solids by the Particle Data Group [30]. The number of tracks, $N$, intercepted by planes of sampling material separated by a distance $x$, can then be obtained through the previously calculated total track length $T$ and is also equivalent to the energy of the incident particle divided by the energy lost $(\delta E)$ by a minimum ionizing particle while traversing a calorimeter sampling/absorber material layer of thickness $x$ :

$$
N=\frac{E}{\epsilon} \frac{X_{o}}{x}=\frac{E}{\delta E} .
$$

Assuming that crossings of sampling material are independent (each track resulting in response in only one sampling material layer) and that their number follows a normal distribution, a parameterization of resolution due only to sampling fluctuations is proposed as Equation (7) ${ }^{5)}$ :

$$
\left(\frac{\sigma(E)}{E}\right)_{\text {sampling }} \simeq \frac{1}{\sqrt{N}}=\sqrt{\frac{\delta E}{F(z) E}}=3.2 \% \sqrt{\frac{\epsilon(M e V)}{F(z)}} \times \sqrt{\frac{t}{E(G e V)}},
$$

where the factor $F(z)$ is the fractional amount of track length which is actually seen by the calorimeter's sampling area and is pendent from the variable $z$ which accounts for shortening of track lengths due to the cut-off energy $\left(E_{c}\right)$ or minimum energy sensitivity of the sampling medium. An approximation for $F(z)$ is given in the form of a numerical calculation done by Richards and Nordheim [22, 25]:

$$
F(z) \simeq e^{z}\left[1+z \ln \left(\frac{z}{1.526}\right)\right]
$$

where $z=4.58(Z / A)\left(E_{c} / \epsilon\right)$. Equation (8) is valid within $10 \%$ for $z \leq 0.3[25]$ ( $z$ is 0.14 and 0.32 for $\mathrm{Cu}$ and $\mathrm{Pb}$, respectively). We used Monte Carlo simulations of our calorimeter prototypes to determine a cut-off for minimum energy sensitivity of the Cherenkov-fiber calorimeter $[4,13,15]$. For quartz and PMMA, there is little or no Cherenkov radiation by charged particles at low velocities $(\beta=v / c<0.65)$. However, this threshold should not be taken as an energy cut-off since Cherenkov photon production as a function of particle velocity drops very quickly for particles below $\beta=0.99$. We chose a cut-off energy for the

5) The $3.2 \%$ constant suddenly popping out of Equation (7) takes into account the conversion of $\epsilon$ from $\mathrm{MeV}$ into $\mathrm{GeV}$, brought outside the radical, and the conversion of the total fraction into percent $\left(100 \% \times \sqrt{10^{-3}}=3.2 \%\right)$. 
Cherenkov-fiber calorimeter by determining the shower track energy above which $95 \%$ of all detected Cherenkov photons are radiated and result in detector response. Below this limit $\left(E_{c}=1.31 \mathrm{MeV}\right.$, corresponding to a particle with $\left.\beta=0.96\right)$, Cherenkov photon production resulting in detector response drops off rapidly; more than $99 \%$ of all detected photons are radiated by charged particles with energies greater than $1 \mathrm{MeV}$. Using this value of $E_{c}$, Equation (8) gives $F(z)=0.69$ for lead and $F(z)=0.77$ for copper, reflecting the fact that more tracks go unobserved by the calorimeter's sampling material when higher $Z$ absorber materials are used.

This approximation by Amaldi for parameterizing the factor $R$ of Equation (4) did not agree with certain experimental results and he made further efforts to arrive at a correction which considers that shower tracks at large angles result in longer pathlengths in the sampling medium causing further sampling fluctuations which affect energy resolution. A shower particle with angle $\theta$ appears to the sampling medium to have a track length $t / \cos \theta$. Equation (7) is then corrected for these high angle tracks by an average factor of $\left(\cos <\theta_{\text {show }}>\right)^{-1 / 2}$. The average of the angle $\theta_{\text {show }}$ for electromagnetic shower tracks depends directly on the ratio $E_{s} / \epsilon$ (through the definition of the Molière radius: $\left.R_{M}=X_{o} E_{s} / \epsilon\right)$ and a quantitative relation was determined though the use of Monte Carlo calculations by Amaldi to be [25]:

$$
<\theta_{\text {show }}>\simeq\left(\frac{E_{s}}{\pi \epsilon}\right),
$$

where $E_{s}$ is the usual constant appearing in multiple scattering theory [22, 30], normally taken to be $E_{s}=21 \mathrm{MeV}$. For example, considering a sampling calorimeter consisting of 3 $\mathrm{mm}$ lead absorber layers with $0.5 \mathrm{~mm}$ of scintillator plates in between, this average angle $\left.<\theta_{\text {show }}\right\rangle$ would be $52^{\circ}\left(19^{\circ}\right.$ for the same configuration but copper absorber $)$ resulting in increased detector response.

However, optical fiber calorimeters collecting Cherenkov light are not sensitive to shower tracks in the same way as sampling calorimeters which depend on isotropically distributed scintillation light. A calorimeter with fibers at $45^{\circ}$ is more sensitive to shower tracks whose angles are closer to the incident particle direction [11, 15]. In addition, Cherenkov light cones from tracks at various angles are only partially collected by the fiber and the fraction collected varies depending on the incident angle and impact distance from the fiber central axis. Therefore, Amaldi's correction for increased tracks lengths is inappropriate for a Cherenkov-fiber calorimeter. We performed Monte Carlo studies on the electromagnetic Cherenkov-fiber prototypes measured and previously described in order to determine the mean track length of shower particles through fiber core material. It was found that the mean track length through fiber core material for shower particles which crossed any part of fiber core material was within $5 \%$ of the fiber core diameter for both lead and copper absorber materials. Our simulations showed that a correction such as that proposed by Amaldi for $\mathrm{dE} / \mathrm{dx}$ based calorimeters then need not be considered for the Cherenkov-fiber case.

However, it should be taken into account that a significant (up to 25\%) fraction of the sampling material zone in between absorber layers can be considered as dead space due to the circular cross-section of fibers and cladding thickness. This dead space will substantially reduce the total shower track lengths to which the calorimeter is sensitive. In addition, the previously mentioned effect of shower tracks which are outside the acceptance angle of fibers is a major contributor to sampling fluctuations in the Cherenkov-fiber calorimeter case. 
Table 4: $F_{\text {obs }}$ (as calculated by Equations (11) and (12)), the average fraction of shower tracks to which a $45^{\circ}$ Cherenkov-fiber calorimeter is sensitive as a function of fiber numerical aperture and for lead and copper absorber materials.

\begin{tabular}{ccc}
\hline $\begin{array}{c}\text { Absorber } \\
\text { Type }\end{array}$ & $\begin{array}{c}\text { Fiber } \\
\text { NA }\end{array}$ & $F_{\text {obs }}$ \\
\hline Lead & 0.22 & 0.19 \\
Lead & 0.37 & 0.35 \\
Lead & 0.40 & 0.38 \\
Lead & 0.51 & 0.50 \\
Copper & 0.22 & 0.12 \\
Copper & 0.37 & 0.33 \\
Copper & 0.40 & 0.38 \\
Copper & 0.51 & 0.58 \\
\hline
\end{tabular}

The fractional volume of sampling material within a fiber layer, $F_{\text {core }}$, is easily arrived at knowing the exact fiber core and cladding dimensions (area of core material divided by the square of the total fiber diameter). The average fraction of tracks which arrive at such angles that they radiate Cherenkov photons which are ultimately observed, $F_{\text {obs }}$, can be estimated by considering the fraction of capturing solid angle of a given fiber with respect to the solid angle between the average angle of shower particles, $\left\langle\theta_{\text {show }}\right\rangle$, given by Amaldi in Equation (9) and the fiber axis angle of $45^{\circ}$. This angle, $\alpha(\theta, \varphi)$, is given through vector analysis to be:

$$
\alpha=\arccos \left(\frac{\sqrt{2}}{2}\left(\sin <\theta_{\text {show }}>\cos \varphi+\cos <\theta_{\text {show }}>\right)\right),
$$

where $\theta$ is the polar angle and $\varphi$ is the azimuthal angle. The ratio of solid angles can then be approximated by:

$$
F=\frac{N A^{2}}{\sin ^{2} \alpha},
$$

for $\arcsin (N A) \leq \alpha$ and $\mathrm{F}=1$ otherwise. The observed fraction, $F_{o b s}$, can then be estimated by the average of the integral of $F$, around $2 \pi$.

$$
F_{o b s}=\frac{1}{2 \pi} \int_{0}^{2 \pi} F d \varphi
$$

Various $F_{o b s}$ as calculated by Equations (11) and (12) for a number of different fiber numerical apertures and for lead and copper absorbers are shown in Table 4. Clearly the fraction $F_{o b s}$ as calculated by Equation (12) follows closely the fibers' numerical aperture although some difference is noted between lead and copper absorber types. This is illustrated more clearly in Fig. 4 where $F_{o b s}$ is plotted as a function of numerical aperture for both lead (Fig. 4a) and copper (Fig. 4b) absorber types. A fibers' numerical aperture is itself a gauge of its capturing power and could also be used as an approximation for $F_{\text {obs }}$, but in that case no correction would be made for the $\mathrm{Z}$ of the absorber material used.

Note that Fig. 4 (b) shows the fraction $F_{o b s}$ for copper to be defined as 1 for numerical apertures greater than 0.9 . The observed fraction, $F_{o b s}$, for copper is slightly less than that for lead below $\mathrm{NA}=0.4$ but increases rapidly above this value. The greater lateral shower spread for lower $\mathrm{Z}$ materials (dictated by the Molière radius) results in a greater fraction of observed tracks only when the capturing power of the fiber light guide is large. 


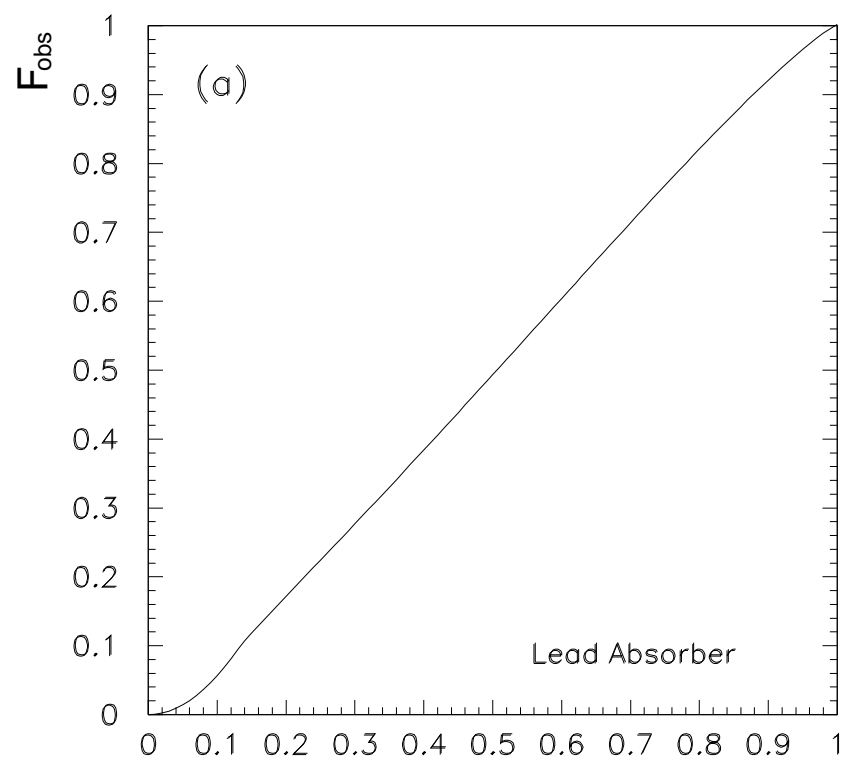

Fiber Numerical Aperture

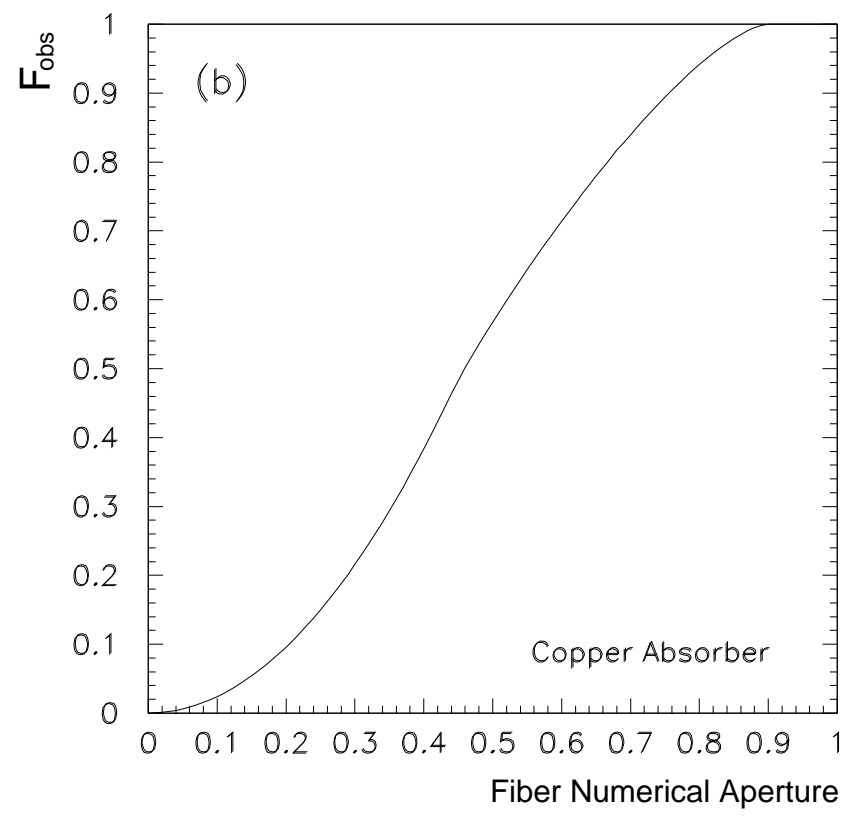

Figure 4: $F_{\text {obs }}$ (as calculated by Equations (11) and (12)) as a function of fiber numerical aperture for lead (a) and copper (b) absorber materials. 
A feel for the physical meaning of $F_{o b s}$ can be gained by considering the limiting case of an optical fiber with a core material whose index of refraction is $n_{\text {core }}=\sqrt{2}$ and with cladding refractive index $n_{\text {clad }}=1$. The numerical aperture of this fiber is then equal to one and, as a result, $F_{\text {obs }} \sim 1$ regardless of the absorber material used. For this case the photon capture angle $\left.{ }^{6}\right)$ is $\theta_{\text {capt }}=45^{\circ}$ and at least some fraction of Cherenkov light from nearly all shower particles (regardless of their trajectories) will be collected if both ends of the fiber are read out or one end is mirrored. $F_{o b s}$ should then be corrected by a factor taking into account $r$, the reflectivity of the mirror opposite the readout end of the fiber. Such a fiber would cause the angular response of a Cherenkov-fiber calorimeter to follow that of a scintillation-based calorimeter where some fraction of the isotropically generated photons are collected regardless of shower track angle.

The calculations presented assume photodetectors at both fiber ends or mirrors opposite from the readout ends. For those calorimeters which have a photodetector at one end of the fiber and no reflective surface at the other end, one would expect an additional loss of detected shower tracks. Measurements of the angular response of Cherenkov-fiber calorimeters exist $[12,16]$. This information can be used to quantitatively determine the extent of "up"/ "down" shower bias existing for the Cherenkov-fiber calorimeter. One such measurement was performed on a Cherenkov-fiber calorimeter prototype (Proto-L). The geometry of Proto-L consisted of fibers arranged in a matrix of lead and sufficiently large so as to contain $8 \mathrm{GeV}$ electromagnetic showers regardless of incident angle. The fibers used for Proto-L were $\mathrm{NA}=0.51$. An angular scan was performed on Proto-L (described in detail in reference [16]) using $8 \mathrm{GeV}$ electrons and the result is shown in Fig. 5. The impacting beam angle was varied by horizontally rotating the calorimeter from $0^{\circ}$ to $150^{\circ}$. The longitudinal length of the module presented to the beam direction a maximum of 35 radiation lengths $\left(\right.$ at $0^{\circ}$ ) and a minimum of 28 radiation lengths (at $90^{\circ}$ ), sufficient to longitudinally contain $99 \%$ of the energy coming from $98 \%$ of all $8 \mathrm{GeV}$ electron showers. The ratio of responses between particles incident at $135^{\circ}$ (down, away from the photodetector) and $45^{\circ}$ (up, towards the photodetector) was measured to be 0.3 . This ratio of responses, though, is directly dependent on the numerical aperture of the fibers used.

An angular response scan (using $4 \mathrm{GeV}$ electrons) was also performed on a different Cherenkov-fiber prototype constructed by an ITEP group [12]. This prototype was similar in geometry to Proto-L but was filled with $\mathrm{NA}=0.22$ fibers and used copper as absorber material. A complete angular scan $\left(>90^{\circ}\right)$ was not performed on the ITEP prototype but the response ratio $R_{90^{\circ}} / R_{45^{\circ}}$ was measured to be 0.13 [12]. From the angular scan for Proto-L (Fig. 5) one can see that the ITEP prototype's angular response ratio, $R_{135^{\circ}} / R_{45^{\circ}} \simeq R_{\text {down }} / R_{u p}$ will be slightly less than that for the ratio $R_{90^{\circ}} / R_{45^{\circ}}$. However, it should be noted that outer dimensions of the ITEP module caused its electromagnetic shower containment for electrons impacting at $90^{\circ}$ (presenting $9 X_{\circ}$ to incoming electrons) to be less than that for electrons impacting at $45^{\circ}$ (presenting $12 X_{\circ}$ ) and the ratio, $R_{90^{\circ}} / R_{45^{\circ}}$, is likely to be slightly greater than the measured value of 0.13 .

The angular response measurements for these two prototypes then leads to a NA dependent estimation for the sum of responses from fiber directions "up" and "down": $R_{\text {up }}+R_{\text {down }} \simeq 0.63+0.37 \times N A$.

A final estimation for the fraction of tracks lengths which produce detector response

6) The limiting angle (between an incident photon and the perpendicular to the core/cladding interface) for total internal reflection: $\theta_{\text {capt }}=\arcsin \left(n_{\text {clad }} / n_{\text {core } e}\right)$. 


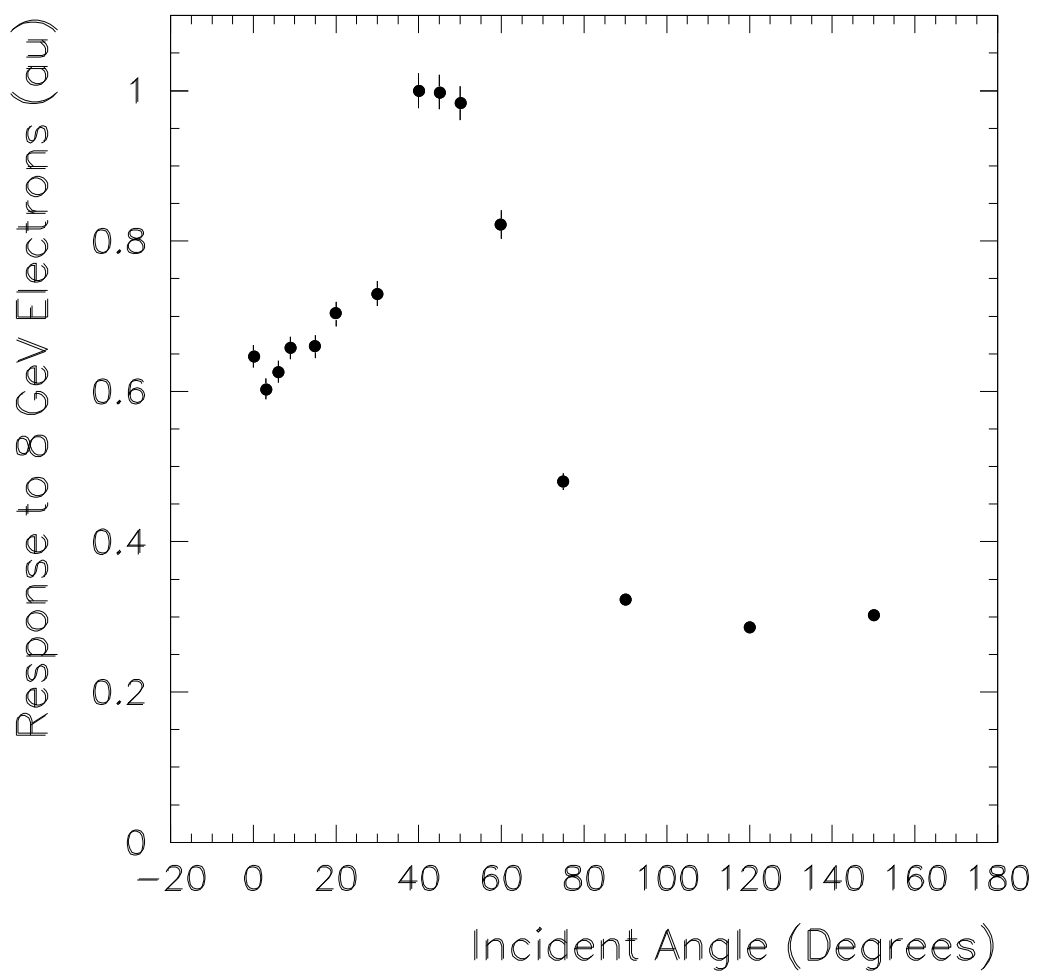

Figure 5: Normalized response of Proto- $\mathrm{L}$ to $8 \mathrm{GeV}$ electrons as a function of incident particle angle.

in the up and down fiber ends can then be written as:

$$
F_{\text {obs effective }}=F_{\text {obs }}\left(R_{u p}+R_{\text {down }} r\right) \simeq F_{\text {obs }}(0.63+0.37 N A \times r)
$$

where $r$ is the fractional reflectivity of mirrors applied to the fiber end faces in the "down" direction, opposite the readout devices. The prototypes constructed so far and described here had no such reflective mirror on their fiber ends opposite the readout phototubes $(r=0)$ and Equation (13) relates the fact that a maximum of only $63 \%$ of all shower tracks which radiated Cherenkov light in fiber core material did so in a manner such that captured photons were reflected towards the photodetector. This simple expression safely ignores the small influence of the numerical aperture on $R_{u p}$ (for the $45^{\circ}$ geometry discussed here).

Applying the correction factors $F_{\text {core }}$ and $F_{o b s}$ to Equation ( 7 ) gives a final estimation for the inverse square root of the number of tracks which cross calorimeter sampling material producing signal:

$$
\begin{aligned}
& \left(\frac{\sigma(E)}{E}\right)_{\text {sampling }} \simeq \frac{1}{\sqrt{N}}=\sqrt{\frac{\delta E}{F(z) F_{\text {core }} F_{\text {obs }}(0.63+0.37 N A \times r) E}} \\
& \simeq 3.2 \% \sqrt{\frac{\epsilon(M e V)}{F(z) F_{\text {core }} F_{\text {obs }}(0.63+0.37 N A \times r)}} \times \sqrt{\frac{t}{E(G e V)}} .
\end{aligned}
$$


Table 5: Pertinent correction factors used for the calculation of degradation in energy resolution due to sampling fluctuations as calculated using Equation (14).

\begin{tabular}{lccccc}
\hline Prototype & $\begin{array}{c}t \\
\text { eq. }(3)\end{array}$ & $\begin{array}{c}F(z) \\
\text { eq. }(8)\end{array}$ & $F_{\text {core }}$ & $\begin{array}{c}F_{\text {obs }} \\
\text { eq. }(12)\end{array}$ & $\begin{array}{c}R(\%) \\
\text { eq. }(4)\end{array}$ \\
\hline Proto-Cu1.5 & $\mathbf{0 . 1 5}$ & $\mathbf{0 . 7 7}$ & 0.74 & $\mathbf{0 . 5 8}$ & 31.5 \\
Proto-Cu3 & $\mathbf{0 . 3 0}$ & $\mathbf{0 . 7 7}$ & 0.74 & $\mathbf{0 . 5 8}$ & 31.5 \\
Proto-Pb3 & $\mathbf{0 . 7 6}$ & $\mathbf{0 . 6 9}$ & $\mathbf{0 . 7 4}$ & $\mathbf{0 . 5 0}$ & 21.6 \\
Proto-3 & $\mathbf{0 . 7 6}$ & $\mathbf{0 . 6 9}$ & $\mathbf{0 . 6 7}$ & $\mathbf{0 . 3 5}$ & 27.1 \\
Proto-4 & $\mathbf{0 . 7 6}$ & $\mathbf{0 . 6 9}$ & $\mathbf{0 . 5 5}$ & $\mathbf{0 . 3 8}$ & 28.7 \\
Proto- $\mathrm{R}$ & $\mathbf{0 . 7 6}$ & $\mathbf{0 . 6 9}$ & $\mathbf{0 . 6 3}$ & $\mathbf{0 . 1 9}$ & 38.0 \\
Proto-Cu20 & 1.98 & $\mathbf{0 . 7 7}$ & $\mathbf{0 . 7 4}$ & $\mathbf{0 . 5 8}$ & 31.5 \\
\hline
\end{tabular}

Table 6: Estimated degradations in energy resolution due to sampling fluctuations as calculated using Equation (14). An estimation using measured energy resolution is also shown for comparison.

\begin{tabular}{|c|c|c|c|}
\hline Prototype & $\begin{array}{c}\left(\frac{\sigma(E)}{E}\right)_{p . e . ~ s t a t i s t i c s} \\
(\% / \sqrt{E}) \\
\text { eq. }(2)\end{array}$ & $\begin{array}{l}\left(\frac{\sigma(E)}{E}\right)_{\text {sampling }} \\
(\% / \sqrt{E}) \\
\text { via eq. }(2)\end{array}$ & $\begin{array}{l}\left(\frac{\sigma(E)}{E}\right)_{\text {sampling }} \\
(\% / \sqrt{E}) \\
\text { eq. }(14)\end{array}$ \\
\hline Proto-Cu1.5 & $13.2 \pm 0.6$ & $21.0 \pm 1.0$ & $12.2 \pm 2.8$ \\
\hline Proto-Cu3 & $18.5 \pm 0.9$ & $21.9 \pm 1.7$ & $17.3 \pm 3.9$ \\
\hline Proto-Pb3 & $26.8 \pm 3.1$ & $21.1 \pm 7.8$ & $18.8 \pm 4.3$ \\
\hline Proto-3 & $25.1 \pm 2.5$ & $26.6 \pm 6.7$ & $23.7 \pm 5.8$ \\
\hline Proto-4 & $28.0 \pm 3.2$ & $31.1 \pm 8.2$ & $25.0 \pm 6.0$ \\
\hline Proto-R & $24.8 \pm 2.5$ & $34.3 \pm 5.1$ & $33.1 \pm 7.1$ \\
\hline Proto-Cu20 & $45.2 \pm 9.2$ & $50.4 \pm 16.9$ & $44.4 \pm 10.2$ \\
\hline
\end{tabular}

\section{Comparison between calculation and measurement}

Equation (14) was applied to the seven $45^{\circ}$ Cherenkov-fiber electromagnetic prototypes previously described and Table 5 summarizes the most pertinent parameters and correction factors. Table 6 gives the calculated electromagnetic energy resolutions caused by sampling fluctuations. The results of beam tests made on the prototypes to determine contributions to the stochastic term from photoelectron statistics (calculated with Equation (2)) and sampling fluctuations (determined by a quadrature subtraction of the contribution due to photoelectron statistical fluctuations from the measured electromagnetic energy resolution) are also shown for comparison with the calculation from Equation (14). Errors for the determination of $(\sigma(E) / E)_{\text {sampling }}$ using Equation (14) were arrived at by first estimating the errors for each of the parameters used in the equation and then combining these errors using standard error analysis. Values arrived at through Equation (14) are 5\% to $20 \%$ lower than those determined via Equation (2) which we attribute to experimental error in either the measurement of photoelectrons per $\mathrm{GeV}$ or calorimeter energy resolution.

The sole exception to this is Proto-Cu1.5 for which there is a $42 \%$ difference. As pointed out by Amaldi [25], one does not expect that this model should apply for very small values of $t=x / X_{\circ}$, where correlations between the number of crossings detected in successive planes become significant. A limit for the validity of this parameterization can be determined by considering that resolution degradations as low as that for a ho- 
mogeneous lead glass calorimeter can be obtained by a sampling calorimeter in which $\epsilon t \simeq 1$, i.e. $t \simeq 1 / \epsilon$ corresponding to $t=0.14$ for lead and $t=0.05$ for copper. At these limits the calorimeter resembles a homogeneous one and when considering calorimeters with $t$ values which approach these limits, alternative parameterizations must be applied. Equation (14) correctly describes electromagnetic energy resolution due to sampling fluctuations for $t \geq 0.3$.

\section{Conclusions}

A parameterization has been derived for the contribution of sampling fluctuations to the degradation of electromagnetic energy resolution of Cherenkov-fiber calorimeters. Results obtained from this parameterization agree well with values deduced from measurements of the energy resolution of a number of different prototypes, after deduction of degradations due to photoelectron statistics. Due to the fundamental assumptions involved, the parameterization's region of applicability ends where the sampling calorimeter begins to resemble a homogeneous one in terms of response.

This model of the energy resolution stochastic term would indicate an absolute, theoretical limit of $\sim 5 \% / \sqrt{E}$ (a homogeneous Cherenkov-fiber calorimeter using NA=1 fibers and read out by an ideal photodetector). A more practical and easily obtained limit, proven by the already existing Cherenkov-fiber prototypes, would be $\simeq \sigma / \sqrt{E} \sim 20 \% / \sqrt{E} \oplus 0 \%$.

\section{$6 \quad$ Acknowledgements}

The authors would like to express their thanks to the CERN Experimental Areas (EA) group, without whose support and high level of professionality this work would not have been possible. The Florida State University group gratefully acknowledges financial support by the U.S. Department of Energy (grant number DE-FG05-87ER40319). The authors are also indebted to the technical expertise of R. Glassman of CRN Strasbourg, France.

\section{References}

[1] C. Baglin et al., IEEE NSS, Orlando, USA, 1992.

[2] B. Grosdidier, Université Louis Pasteur Doctoral Thesis, CRN 92-45, order number 1336, 10 July, 1992.

[3] P. Gorodetzky et al., Radiation Physics and Chemistry, (Pergamon Press, Oxford, U.K.) 41 nos. 1/2, (1993) p. 253, eds. R. Clough and K.F. Johnson.

[4] D. Lazic, Université Louis Pasteur Doctoral Thesis, CRN 93-38, order number 1534, 28 May, 1993.

[5] A. Contin et al., CERN/DRDC/94-4, January 5, 1994.

[6] K.F. Johnson et al., Proceedings of the Fourth International Conference on Calorimetry in High Energy Physics, (World Scientific Pub. Co., Singapore, 1994) p. 438, eds. A. Menzione and A. Scribano.

[7] P. Gorodetzky et al., ref. [6], p. 433.

[8] P. Gorodetzky et al., ref. [6], p. 462.

[9] A. Musso et al., ref. [6], p. 457.

[10] B. Christie et al., RHIC note 13, STAR note 175, Brookhaven, May 13th, 1994.

[11] A. Contin et al., Nucl. Instr. and Meth. A357 (1995) pp. 369-379.

[12] V. Gavrilov et al., Nucl. Instr. and Meth. A360 (1995) pp. 237-239.

[13] M. Marino et al., Nucl. Instr. and Meth. A357 (1995) pp. 380-385. 
[14] M. Lundin et al., Nuclear Physics B (Proc. Suppl., eds. E. Borchi, S. Majewski, J. Huston, A. Penzo and P.G. Rancoita) 44 (1995) pp. 168-172.

[15] P. Gorodetzky et al, Nucl. Instr. and Meth. A361 (1995) pp. 161-179.

[16] D. Lazic et al., Nucl. Instr. and Meth. A367 (1995) pp. 271-275.

[17] E. Chiavassa et. al., Nucl. Instr. and Meth. A367 (1995) pp. 267-270.

[18] J. Britz et al., CERN/LHCC 95-27, LDRB Status Report/RD40, 15 April 1995.

[19] M. Lundin, Université Louis Pasteur Doctoral Thesis, CRN 95-30, order number 2058, 15 May, 1995.

[20] O. Ganel and R. Wigmans, Nucl. Instr. and Meth. A365 (1995) pp. 104-116.

[21] J. Proudfoot, ref. [3], p. 1.

[22] B. Rossi, High Energy Particles, Prentice-Hall, New York (1952).

[23] G. Fisher, Nucl. Instr. and Meth. 156 (1978) p. 81.

[24] V. Radeka and W.J. Willis, Nucl. Instr. and Meth. 120 (1974) p. 221.

[25] Ugo Amaldi, Physica Scripta, Vol. 23 (1981) 409.

[26] D.E. Groom, Proceedings of the II International Conference on Calorimetry in High Energy Physics, pp. 376-381, Capri, Italy, 14-18 October 1991, ed. by A. Ereditato, World Scientific (1992).

[27] S.L. Stone et al., Nucl. Instr. and Meth. 151 (1978) 387.

[28] J. del Peso and E. Ros, DESY 88-094, July 1988.

[29] M.J. Berger and S.M. Seltzer, "Tables of Energy Losses and Ranges of Electrons and Positrons," NASA-SP-3012 (Washington, DC 1964).

[30] Review of Particle Properties, Phys. Rev. D, 1 August 1994, Vol. 50, Part I. 